\title{
Critical properties of the band-insulator-to-Mott-insulator transition in the strong-coupling limit of the ionic Hubbard model
}

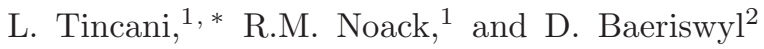 \\ ${ }^{1}$ Fachbereich Physik, Philipps Universität Marburg, D-35032 Marburg, Germany \\ ${ }^{2}$ Département de Physique, Université de Fribourg, CH-1700 Fribourg, Switzerland
}

(Dated: January 14, 2008)

\begin{abstract}
We investigate the neutral-to-ionic insulator-insulator transition in one-dimensional materials by treating a strong-coupling effective model based on the ionic Hubbard model using the densitymatrix renormalization group and finite-size scaling. The effective model, formulated in a spin-one representation, contains a single parameter. We carry out an extensive finite-size scaling analysis of the relevant gaps and susceptibilities to characterize the two zero-temperature transitions. We find that the transition from the ionic band-insulating phase to an intermediate spontaneously dimerized phase is Ising, and the transition from the dimerized phase to the Mott-insulating phase is Kosterlitz-Thouless, in agreement with the field-theory-based predictions.
\end{abstract}

PACS numbers: 71.10.-w, 71.10.Fd, 71.10.Hf, 71.30.+h

Electrons in solids are subject to both a single-particle potential and the Coulomb interaction. A wealth of interesting phenomena can occur when the form of the singleparticle potential deviates from that of the ideal crystal due to, for example, structural transitions, lattice vibrations, or defects or impurities. A simple Hamiltonian that incorporates the combined effects of interactions and reduced translational symmetry in a particularly transparent manner is the ionic Hubbard model (IHM), in which the single-particle energy alternates between neighboring sites. This model was introduced by Nagaosa and Takimoto $\underline{1,2.3}$ to describe the neutral-ionic transition observed by Torrance et al. in mixed-stack organic chargetransfer compounds. $\underline{\underline{4}}$ In a mixed stack of donor (D) and acceptor (A) molecules, the neutral phase corresponds to a uniform and neutral distribution of charge, $\mathrm{D}^{0} \mathrm{~A}^{0} \mathrm{D}^{0} \mathrm{~A}^{0}$, and the ionic phase to an alternation of positive and negative charges, $\mathrm{D}^{+} \mathrm{A}^{-} \mathrm{D}^{+} \mathrm{A}^{-}$. The insulating behavior in the neutral phase originates from the Coulomb interaction between electrons, i.e., the Mott mechanism, whereas the ionic phase is essentially a band insulator. Recently, the neutral-ionic transition has been observed in organic charge-transfer compounds close to zero temperature, motivating interest in it as a pure quantum phase transition $\underline{\underline{5}}$

A different class of quasi-one-dimensional materials in which a similar charge disproportionation occurs is that of the halogen-bridged transition-metal complexes, whose structure is formed by a backbone of of alternating metal and halogen atoms $\underline{\underline{6}}$ In these MX-chain compounds (or in the related MMX materials ${ }^{7}$ ), a spontaneous breaking of the translational symmetry occurs due to the dimerization of the halogen sublattice, XMX-M$\mathrm{XMX}-\mathrm{M}$. The differing distances of the halogen ions from the neighboring metal ions give rise to a two-fold alternation in the energy of the $d$ levels.

The Hamiltonian of the ionic Hubbard model can be grouped into three terms, a one-dimensional nearestneighbor hopping term with matrix element $t$, an on-site Coulomb repulsion of strength $U$, and an ionic alternat- ing potential of depth $\Delta$,

$$
\hat{H}=\hat{H}_{t}+\hat{H}_{U}+\hat{H}_{\Delta},
$$

with

$$
\begin{gathered}
\hat{H}_{t}=t \sum_{i=1, \sigma}^{L-1}\left(\hat{c}_{i \sigma}^{\dagger} \hat{c}_{i+1 \sigma}+\hat{c}_{i+1 \sigma}^{\dagger} \hat{c}_{i \sigma}\right), \\
\hat{H}_{U}=\frac{U}{2} \sum_{i=1, \sigma}^{L} \hat{n}_{i \sigma} \hat{n}_{i-\sigma},
\end{gathered}
$$

and

$$
\hat{H}_{\Delta}=\frac{\Delta}{2} \sum_{i=1, \sigma}^{L}(-1)^{i} \hat{n}_{i \sigma} .
$$

Here $\hat{c}_{i \sigma}^{\dagger}\left(\hat{c}_{i \sigma}\right)$ are the usual creation (annihilation) operators on site $i$ for an electron of spin $\sigma$ and $\hat{n}_{i \sigma}=\hat{c}_{i \sigma}^{\dagger} \hat{c}_{i \sigma}$. Without the ionic potential, $\Delta=0$, the model reduces to the one-dimensional Hubbard model, whose behavior is well understood. $\stackrel{8}{-}$ Although the overall physics described by the ionic Hubbard model is now fairly well known, many details of the transition are still unclear. The general behavior in the ground state is summarized in the schematic ground-state phase diagram shown in Fig. 1, When $\Delta \gtrsim U$, the system is a band insulator (BI) and has both a charge and spin gap. When $\Delta \lesssim U$, the system is a critically antiferromagnetic Mott insulator (MI) with a charge gap and gapless spin excitations. These two phases are separated by two continuous phase-transition lines within which there is a spontaneously dimerized insulating phase (SDI) of width of order $t$, i.e., a phase with both spin and charge gaps as well as with long-range bond dimer order.

In order to understand the origin of the phases, let us first examine what happens in the atomic limit, $t=0$, which can be easily treated. For $U>\Delta$ and at half 


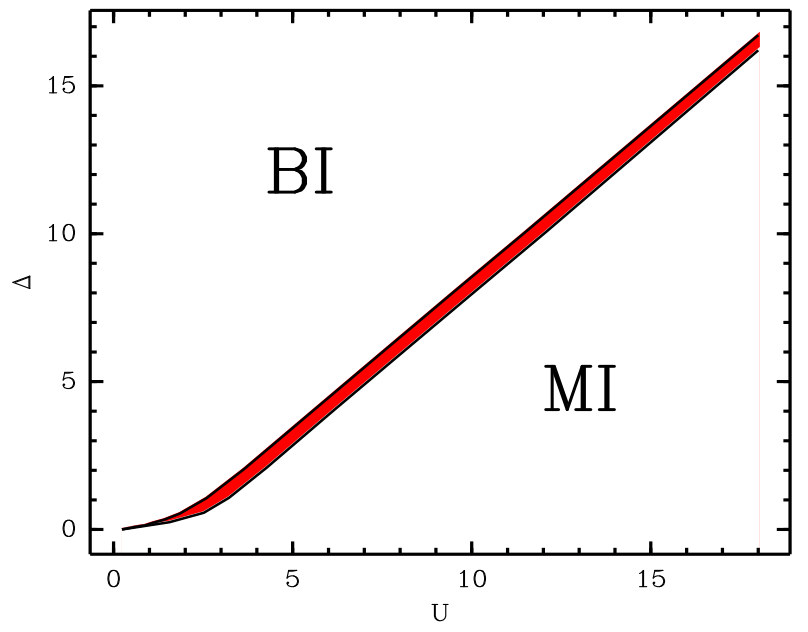

Figure 1: (Color online) Ground-state phase diagram of the ionic Hubbard model. Location of the phase boundaries is approximate, but drawn to scale according to values from Refs. 9 and 10. The (red) shaded intermediate region designates a spontaneously dimerized insulating phase (SDI).

filling, there is no double occupancy in the ground state, which consists of a series of singly occupied sites with energy $\pm \Delta / 2$ so that the entire system has energy $E=$ 0 . For $U<\Delta$, double occupancy is favorable, and the ground state consists of doubly occupied sites at energy $U-\Delta$ alternating with empty sites, so that the energy of the system is $L(U-\Delta) / 2$. At $(U-\Delta) \rightarrow 0$, a level crossing of two configurations occurs and there is a firstorder transition at $U=\Delta$.

Turning on the hopping term leads to more subtle behavior in the vicinity of the transition. In the noninteracting limit, $U=0$, the Hamiltonian is diagonal in momentum space. It follows that the ionic term, $\Delta$, opens a charge and a spin gap, and the two gaps have the same value. Correspondingly, spin-spin and chargecharge correlations decay exponentially. The scenario does not change with the inclusion of a weak interaction $\hat{H}_{U}$; the electrons tend to doubly occupy sites with lower potential, and the system remains a band insulator.

In the large- $U$ limit, the double occupancy can be treated perturbatively and the low-energy physics of the IHM is described by an effective spin one-half Heisenberg model $, 1,11,12$ It is important to note that this effective model restores translational invariance, and that the charge and spin sectors are completely separated. The system has gapless spin excitations and critical spin-spin correlations, while the charge gap, in contrast, scales as $U$ for large $U$. This description is robust for a wide range of parameters in the strong coupling limit, but fails close to the transition line because perturbation theory breaks down in the critical regime $\underline{\underline{1}}$ In fact, there are analytical and numerical indications that show that higher-order spin excitations mix into the charge degrees of freedom everywhere in the MI phase $\frac{9,10,13}{13}$

A few years ago, Fabrizio, Gogolin and Nersesyan proposed a new, interesting scenario based on fieldtheoretical arguments. $\underline{\underline{14}}$ They argued that two quantum phase transitions occur, an Ising transition between the band insulator and an intermediate spontaneously dimerized phase, followed by (for increasing $U / \Delta$ ) a KosterlitzThouless transition (KT) between the dimerized phase and the Mott insulator. This scenario is based on an argument in which the transition is approached, on the one hand, from the MI limit and, on the other hand, from the BI limit. The authors consider the weak-coupling case, $(U, \Delta)<<t$, and use standard bosonization. The Hamiltonian then consists of three parts, a first term depending only on charge degrees of freedom, a second term involving only spin degrees of freedom and a third term, proportional to $\Delta$, which couples charge and spin degrees of freedom. Starting from the MI phase ( $U$ dominating) with a charge gap but no spin gap, one can integrate out the charge degrees of freedom. This leads to a sineGordon model for the spin degrees of freedom with a positive coupling for $U>U_{c_{2}}$. The coupling term turns negative for $U<U_{c_{2}}$, and therefore $U_{c_{2}}$ corresponds to a KT transition point. A spin gap opens for $U<U_{c_{2}}$ and is attributed to a spontaneously dimerized insulating phase (SDI). Starting from the BI phase ( $\Delta$ dominating), which exhibits both a charge and a spin gap, Fabrizio, Gogolin and Nersesyan calculate spin and bond-order susceptibilities using perturbation theory. A critical value $U_{c_{1}}$ is found where the bond-order susceptibility diverges, while the spin susceptibility remains finite. Thus, $U_{c_{1}}$ must be in a region with a finite spin gap, and it follows that $U_{c_{1}}<U_{c_{2}}$. Close to $U_{c_{1}}$ it is argued that the spin degrees of freedom can be considered to be frozen. This yields a double sine-Gordon Hamiltonian for the charge degrees of freedom, which is known to undergo a quantum phase transition of an Ising type $\stackrel{15}{\underline{15}}$ The order parameter of this transition is the bond order operator, which confirms that the intermediate region, $U_{c_{1}}<U<U_{c_{2}}$, is in a SDI phase $\underline{\underline{16}}$

At least one transition has been found in all numerical work $9,10,11,17,18,19,20,21,22$ published after Ref. 14, although, for the most part, the critical behavior was not characterized. The critical exponents were calculated in Ref. 10, but were found to deviate from the expected two-dimensional-Ising values. However, even confirming that there is a second transition has been a quite difficult task. The two transitions turn out to be very close to one another and, since the transition to the Mott insulator is expected to be a KT transition, it is very difficult to find and characterize using finite-size-scaling studies $\stackrel{10}{\underline{10}}$ For these reasons, studying an effective model characterizing the region of the transition and the intermediate phase is useful.

Another very important subtlety is how to map the gaps from the field-theoretical model onto the original lattice model. In the ionic Hubbard model, the charge gap, the one-particle gap, and the spin gap all behave differently at the transitions. The one-particle gap is related to the charge and spin gaps but is fundamentally 
different because it involves a change of the particle number, while the charge and spin gaps are spectral gaps of excitations into the charge and spin sectors, respectively, only. One way of locating critical points is to examine the smallest energy gap, i.e., the mass gap, as a function of the tuning parameters. The critical point is then the point at which the gap vanishes in the thermodynamic limit.

The remainder of this paper is organized as follows. In Sec. I], an effective spin-one model for the transition is derived via a strong-coupling treatment. In Sec. II the numerical method used to study the model is described. In Sec. III and IV we report the analysis of the band-insulator-to-spontaneously-dimerized insulator and the spontaneously-dimerized-to-Mott insulator transitions, respectively.

\section{EFFECTIVE MODEL}

\section{A. Derivation of the effective Hamiltonian}

In order to investigate the critical behavior of the ionic Hubbard model at half filling, we derive an effective model, formulated in terms of spin-one operators, valid for $(U, \Delta)>>t$. In this limit, the doubly occupied state on the even sites (with on-site potential $\Delta / 2$ ) and the unoccupied state on the odd sites can be projected out. At half filling, a double occupancy on an even site is necessarily associated with a completely unoccupied odd site, and has a cost in energy of $U+\Delta$. This procedure is a second-order strong-coupling expansion with parameter $t /(U, \Delta)$ analogous to that used to derive the $t-J$ model from the Hubbard model. In fact, the resulting model can equivalently be formulated in terms of $t$ - $J$ operators rather than spin-one operators; we feel that the latter formulation is more intuitive for the half-filled system. 23,24 The physical meaning of the spin-one states is as follows: the $S_{z}= \pm 1$ state corresponds to a singly occupied site with a spin- $\frac{1}{2}$ electron with spin up or down, while the $S_{z}=0$ state corresponds to an unoccupied site on the even sites and a doubly occupied site on the odd sites. The mapping of the states of the ionic Hubbard model to those of the effective spin-one model is summarized in Table [.

As we shall see, conservation of particle number leads to a spin exchange process for the spin-one operators that is more restricted than the Heisenberg exchange. Given the mapping of states described above, the effective Hamiltonian can most easily be derived by first expressing the original Hamiltonian in terms of transition operators between the fermionic states (Hubbard operators), then projecting out the states as outlined above, and subsequently writing the Hamiltonian in the reduced state space in terms of spin transition operators. Finally, the transition operators in spin space can be rewritten in terms of spin-one operators $\stackrel{25,26}{ }$ A detailed derivation is given in the appendix. .
Table I: Mapping between the single-site basis states of the ionic Hubbard model $\{|0\rangle,|\uparrow\rangle,|\downarrow\rangle,|d\rangle\}$ with $|d\rangle$ denoting the doubly occupied state, and those of the effective spin-one model $\left\{\left|S^{z}\right\rangle\right\}$.

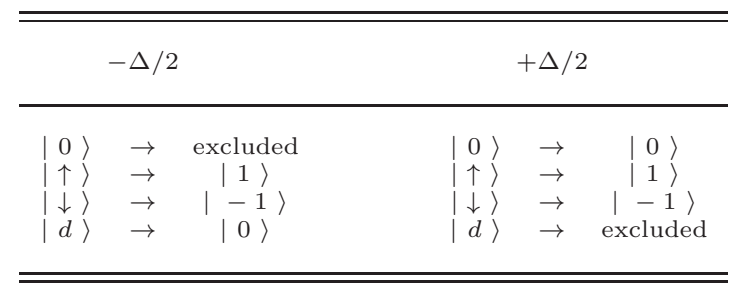

The Hamiltonian for the effective spin-one model can thus be expressed in terms of the usual spin-one operators, yielding $\hat{H}^{e}=\hat{H}_{t}^{e}+\hat{H}_{\varepsilon}^{e}$, with the exchange term

$$
\begin{aligned}
\hat{H}_{t}^{e}=\frac{t}{2} \sum_{i=1}^{L}[ & \left(\hat{S}_{i}^{+} \hat{S}_{i+1}^{-}+\hat{S}_{i}^{-} \hat{S}_{i+1}^{+}\right) \hat{S}_{i+1}^{z} \\
& \left.-\hat{S}_{i}^{z}\left(\hat{S}_{i}^{+} \hat{S}_{i+1}^{-}+\hat{S}_{i}^{-} \hat{S}_{i+1}^{+}\right)\right]
\end{aligned}
$$

and the interaction term governed by the single parameter $\varepsilon=U-\Delta$,

$$
\hat{H}_{\varepsilon}^{e}=-\frac{\varepsilon}{2} \sum_{i=1}^{L}\left[\left(\hat{S}_{i}^{z}\right)^{2}-1\right] .
$$

Note that it is immediately clear from the effective model that the relevant interaction parameter is $\varepsilon=U-$ $\Delta$. For $t=0$, it is clear that there should be a transition at $U \sim \Delta$ because the sign of the $\hat{H}_{\varepsilon}^{e}$ term changes. For $\varepsilon>>t$, the on-site $S^{z}=0$ state is strongly suppressed so that the remaining degrees of freedom, $S^{z}=1$ and $S^{z}=$ -1 , correspond to the localized spin- $\frac{1}{2}$ degrees of freedom of the MI phase of the original model. For $\varepsilon \rightarrow-\infty$, the $S^{z}= \pm 1$ local states are suppressed, leading to a ground state that is a simple product of local $S^{z}=0$ states, which maps to the band insulator. However, the nature of the transition(s) and possible intermediate phases for finite $t$ still needs to be determined. In particular, it is important to investigate whether the behavior in the vicinity of $\varepsilon=0$ agrees with previous numerical results for the ionic Hubbard model, 10,11,22 as well as with fieldtheoretical treatments. 14

Note that the derivation of the effective model can easily be extended to include additional interaction terms that do not break the symmetries of the original model, such as a next-nearest-neighbor Coulomb repulsion. In a similar context, a related effective model was developed some time ago in Ref. 27.

\section{B. Observables}

Since the formulation of the effective model in terms of spin-one operators is a notational convenience rather 
than physical, we are interested in studying observables of the original model. Therefore, it is necessary to translate the observables of the IHM into the language of the spin-one model. The local spin operators map as (small letters: IHM, capital letters: effective model)

$$
\begin{aligned}
& \hat{s}_{i}^{z} \rightarrow \frac{1}{2} \hat{S}_{i}^{z}, \\
& \hat{s}_{i}^{ \pm} \rightarrow \frac{1}{2}\left(\hat{S}_{i}^{ \pm}\right)^{2}, \\
& \hat{S}_{i}^{2} \rightarrow \frac{3}{4}\left(\hat{S}_{i}^{z}\right)^{2},
\end{aligned}
$$

the local charge operators as

$$
\hat{n}_{i} \rightarrow\left\{\begin{array}{rl}
\left(\hat{S}_{i}^{z}\right)^{2} & \mathrm{i}=\text { even } \\
2-\left(\hat{S}_{i}^{z}\right)^{2} & \mathrm{i}=\text { odd }
\end{array}\right.
$$

and total spin and charge operators as

$$
\begin{aligned}
& \hat{s}^{z} \rightarrow \frac{1}{2} \hat{S}^{z}, \\
& \hat{s}^{2} \rightarrow \frac{1}{2} \hat{S}^{z}\left(\frac{1}{2} \hat{S}^{z}+1\right)+\frac{1}{4} \sum_{i, j=1}^{L}\left(\hat{S}_{i}^{-}\right)^{2}\left(\hat{S}_{j}^{+}\right)^{2}, \\
& \hat{N} \rightarrow L+\sum_{i=1}^{L}(-1)^{i}\left(\hat{S}_{i}^{z}\right)^{2} .
\end{aligned}
$$

As we can see, conservation of $s^{z}$ in the IHM leads to conservation of $S^{z}$ in the effective model, with the spin scaled by a factor of one half. However, conservation of the total spin in the IHM does not lead to conservation of total spin for the effective model, which is not $S U(2)$-invariant. In Table we show the mapping of the most important quantities from the original ionic Hubbard model to the effective spin-one model.

\section{Symmetries}

One relevant characteristic of the effective model is the extent to which the symmetries of the original model are preserved or modified. The interaction term $\hat{H}_{\varepsilon}^{e}$ is local, translationally invariant, and depends only on $\left(S^{z}\right)^{2}$, in contrast to the on-site part of the IHM Hamiltonian in Eq. (1). The apparently greater translational symmetry of the effective model is a consequence of the reduction of state space in transforming to the effective model. Note that this is only true at half filling: the quantity $\langle\hat{N}\rangle-L$ (see Eq. 7) is conserved and breaks translational symmetry except at half filling, where it is zero. (Note that the interpretation of the $S^{z}=0$ state is not translationally invariant.) Since the spin-exchange term has the same symmetries as the hopping term in the IHM, the remaining symmetries of the original model are preserved in the effective model. Conserved quantities in the original model, such as the total z-component of the spin, $s_{z}$, the total spin, $s$, and the number of particles, $N$, are still conserved in the effective model, but have different meanings.

\section{NUMERICAL METHOD}

We have investigated the effective model by performing density-matrix renormalization group (DMRG) calculations for different system sizes, from $L=200$ up to 600 sites, with open boundary conditions (OBC) and an even number of sites 28,29 For small chains, boundary effects can be large, depending on the correlation length. Thus, in order to minimize any dispersion due to the edges, $\underline{30}$ Friedel oscillations,,$\frac{31}{20}$ and odd-even effects,,$\frac{32}{2}$ we analyze systems of at least 200 sites. In order to achieve sufficient accuracy, at least 5 sweeps must be performed, with up to 1280 states retained in the last sweep. The maximum system size that can be accurately treated is then approximately 600 sites 33 The maximum discarded weight of the density matrix for the effective model is always less than $10^{-8}$, and is typically zero to within the numerical precision far from the critical points $\stackrel{34}{ }$ In order to calculate ground-state properties, we target the ground state in the $S^{z}=0$ sector; we target both the ground state and the first excited state in the $S^{z}=0$ sector to calculate the 'exciton' gap of the original IHM; and the lowest states in the $S^{z}=1$ and $S^{z}=2$ sectors are needed to calculate the charge and spin gaps, respectively, of the IHM 10

We have repeated the same calculations using the dynamic block-state selection (DBSS) approach, fixing the threshold of maximum quantum information loss to $\chi=10^{-6}$ at each step $\underline{34,35}$ For instance, $m \approx 500$ basis states are enough to correctly describe the ground-state wave function of a system with 500 sites for $\varepsilon=1.23$. However, as we increase $\varepsilon$ the number of states required increases, for example to $m \approx 900$ states for $\varepsilon=2$. For ground states of other symmetry sectors, e.g., the lowest triplet excitation, this number can sometimes be larger when the excited state is delocalized, despite the fact that its Fock subspace is smaller. Nevertheless, since we are interested in only the energy of these states and since measurements are carried out only on the absolute ground state, keeping of the order of a thousand states is usually sufficient.

As the aim of the effective model is to describe the strong-coupling limit of the IHM when $(U, \Delta)>>t$, we have compared results from the effective model to DMRG results for the IHM for $U \simeq \Delta=20 t .10$ All the quantities that we measure: gaps, ionicity, bond order parameter and polarization, are in agreement to within a few percent. 
Table II: Mapping of relevant physical quantities to the effective spin one model.

\begin{tabular}{lll}
\hline \hline Quantity & Ionic Hubbard Model & Effective Spin One Model \\
\hline Ionicity & $I=\frac{2}{L} \sum_{i=1}^{L}(-1)^{i}\left\langle\hat{n}_{i}\right\rangle$ & $I=2-\frac{2}{L} \sum_{i=1}^{L}\left\langle\left(\hat{S}_{i}^{z}\right)^{2}\right\rangle$ \\
Polarization & $P_{e}=\frac{1}{L} \sum_{i=1}^{L} x_{i}\left\langle\hat{n}_{i}\right\rangle$ & $P_{e}=\frac{1}{L} \sum_{i=1}^{L}(-1)^{i} x_{i}\left\langle\left(\hat{S}_{i}^{z}\right)^{2}\right\rangle-\frac{1}{2}$ \\
Bond Order Parameter & $D=\frac{1}{L-1} \sum_{i=1}^{L-1}(-1)^{i}\left\langle\hat{c}_{i}^{\dagger} \hat{c}_{i+1}+\hat{c}_{i+1}^{\dagger} \hat{c}_{i}\right\rangle$ & $D=\frac{1}{L-1} \sum_{i=1}^{L}(-1)^{i}\left[\left\langle\left(\hat{S}_{i}^{+} \hat{S}_{i+1}^{-}+\hat{S}_{i}^{-} \hat{S}_{i+1}^{+}\right) \hat{S}_{i+1}^{z}\right.\right.$ \\
AFM Order & $A=\frac{1}{L} \sum_{i}^{L}(-1)^{i}\left\langle\hat{S}_{i}^{z}\right\rangle$ & $A=\frac{1}{2 L} \sum_{i}^{-}(-1)^{i}\left\langle\hat{S}_{i}^{z}\right\rangle$ \\
\hline \hline
\end{tabular}

\section{BI TO SDI TRANSITION}

In this section we study the first transition between the band-insulator phase and the spontaneously dimerized phase. We have tuned the interaction coupling $\varepsilon$ starting from zero, where the system behaves like a band insulator, increasing it until the first transition point $\varepsilon_{c_{1}}$ is reached. In order to locate the transition point, we have studied the behavior of the singlet and triplet gaps and of the bond order parameter. The two gaps go to zero in the thermodynamic limit at the transition point and subsequently reopen. The value of the bond order parameter, which measures the system's dimerization, changes from zero to a finite value across the transition. The existence of such a transition has been extensively discussed for the IHM. ${ }^{10,22}$ Therefore, we have focused on the characterization of the transition by evaluating its critical exponents explicitly.

The Hamiltonian of the effective model is new and is not evidently related to any known classical model. Therefore, we must first determine the value of the dynamic critical exponent $z$ in order to carry out finite-size scaling. Subsequently, we extract the correlation length exponent $\nu$ from the divergence of the mass gap. Finally, the thermodynamic exponents $\beta, \alpha$ and $\gamma$, which are all related to the free energy density, are obtained by analyzing the divergence of the bond order parameter, the specific heat, and the bond-order susceptibility, respectively.

\section{A. Dynamic critical exponent $z$}

For a quantum system related to a classical model by the transfer matrix, the dynamic critical exponent plays the role of an extra dimension, i.e., $z=1$. In general, space and time correlations can be coupled, and the value of $z$ can be different from one. Therefore, a determination of $z$ is required to obtain and interpret all the remaining critical exponents. First, we identify the mass gap

$$
F(\varepsilon, L)=E_{1}(\varepsilon, L)-E_{0}(\varepsilon, L),
$$

which is the gap that scales to zero most quickly close to the critical point $\underline{36}$ This gap is proportional to $\xi^{-z}$, where the correlation length $\xi$ is limited by the system size $L$. Consequently, the ratio

$$
R_{z}(\varepsilon, N, M)=\frac{F(\varepsilon, N)}{F(\varepsilon, M)} \frac{N}{M}
$$

of the mass gaps for different system sizes behaves as $R_{z}\left(\varepsilon_{c_{1}}, N, M\right) \sim(N / M)^{1-z}$ for $N, M>>1$, and thus depends only on the ratio of system sizes $r \equiv N / M \stackrel{37}{ }$ In Fig. 2(a), we show that all the gap ratios with a particular $r(r=1.5$ in the figure) cross each other at the same point, which is near $R_{z}=1$. The behavior is similar for other values of $r$; we have examined $r=1.2,1.25$, 1.33, and 2. In Fig. 2(b), one can see that curves with different $r$, scaled by the $M=200$ gap, cross $R_{z}=1$ at the same point. Thus, it is clear that all curves cross each other at approximately the same value of $\varepsilon, \varepsilon \approx 1.3$, where $R_{z}(N, M) \approx 1$, consistent with $z=1.38$ In order to carry out the scaling analysis of the critical coupling and other critical exponents, we take $z=1$ in the following subsections.

\section{B. Correlation length exponent $\nu$}

In order to proceed, we next need to calculate the critical value of the coupling in the thermodynamic limit, $\varepsilon_{c_{1}}$. The most efficient and accurate way of doing this is to carry out scaling using the logarithmic mass gap ratio, 39 defined as

$$
R(\varepsilon, L)=\frac{\ln F(\varepsilon, L+2)-\ln F(\varepsilon, L)}{\ln (L+2)-\ln L} .
$$




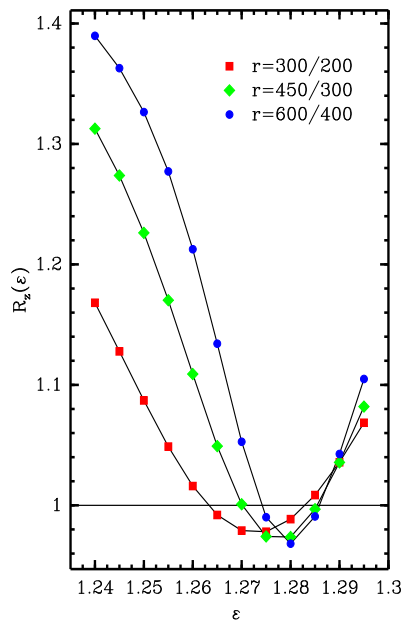

(a)

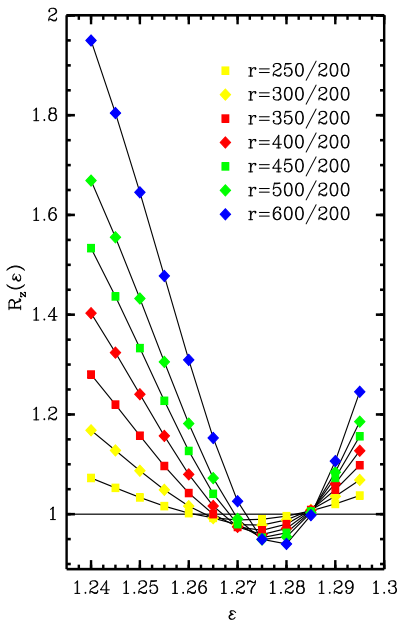

(b)
Figure 2: (Color online) Mass gap ratio $R_{z}$ as function of the coupling $\varepsilon$ for (a) various system sizes $N$ and $M$ and the same ratio $r=1.5$, and (b) mass gap ratio for different $r$ scaled by the $M=200$ gap.

This quantity can be used to define a sequence of pseudocritical points for different system sizes using the criterion $R\left(\varepsilon^{*}, L\right)+1=0$. In Fig. 3(a) we show the behavior of $R\left(\varepsilon^{*}, L\right)+1$ as a function of $\varepsilon$ for various system sizes. The curves of the scaled ratio cross the line at two points, defining two sets of pseudo-critical points, which we designate as $\varepsilon_{a}^{*}(L)$ and $\varepsilon_{b}^{*}(L)$ for the lower and upper crossings, respectively. The finite-size scaling of both series of pseudo-critical points is depicted in Fig. 3(b). All curves are fit with third-order polynomials in $1 / \mathrm{L}$. In the thermodynamic limit, $\varepsilon_{a}^{*}$ and $\varepsilon_{b}^{*}$ converge to the same point to within the accuracy of the extrapolation, confirming that the transition is second order. The finite-size scaling of the position of the minimum in the mass gap provides an alternate way of determining $\varepsilon_{c_{1}}$. This can either be done using the mass gap, Eq. (8), directly, which we designate as $\varepsilon_{m}(L)$, or using the minimum of the mass-gap ratio, Eq. (9), designated as $\varepsilon_{r}(L)$. The extrapolations of positions of the minima, $\varepsilon_{m}$ and $\varepsilon_{r}$ also converge to the same point, providing a confirmation of the consistency and stability of the extrapolation procedure. We obtain the location of the critical point at

$$
\varepsilon_{c_{1}}=1.286(5) \text {. }
$$

We can now estimate the correlation-length exponent using the finite-size version of the Callan-Symanzik $\beta$ function $37,39,40,41$

$$
\beta_{\mathrm{cs}}^{-1}(\varepsilon, L)=\frac{1}{F(\varepsilon, L)} \frac{\partial F(\varepsilon, L)}{\partial \varepsilon},
$$

which has critical behavior

$$
\beta_{\mathrm{cS}}\left(\varepsilon_{c_{1}}, L\right) \sim L^{-\frac{1}{\nu}}
$$

To calculate the exponent $\nu$, we proceed as follows: Given

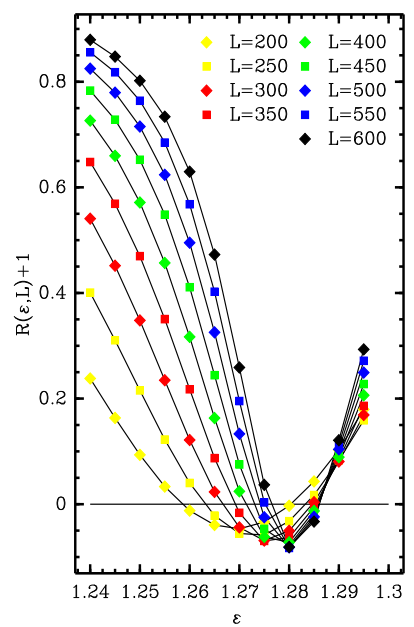

(a)

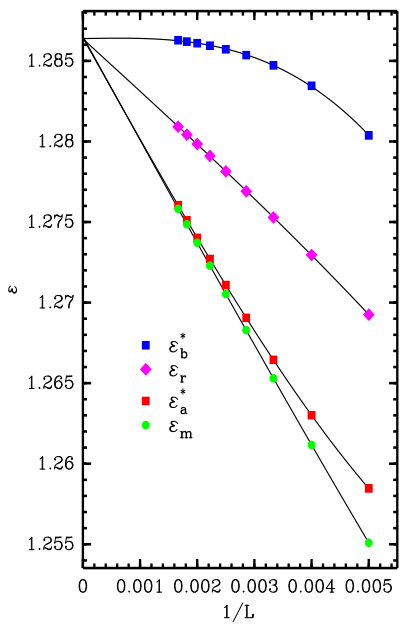

(b)
Figure 3: (Color online) (a) Logarithmic mass gap ratio as a function of $\varepsilon$ for various system sizes and (b) finite-size extrapolations of the critical point using both sequences of pseudo-critical points, as well as the two definitions of the gap minimum. Here $\varepsilon_{m}$ is the position of the mass gap minimum, $\varepsilon_{r}$ the position of the mass-gap ratio minimum, and $\varepsilon_{a}^{*}$ and $\varepsilon_{b}^{*}$ are the upper and lower sequences of pseudo-critical points, respectively. The lines are guides to the eye.

a sequence of pseudo-critical points, $\varepsilon^{*}(L)$, we extrapolate the ratio of the $\beta$-functions for different system sizes

$$
\frac{\beta_{\mathrm{cs}}\left(\varepsilon^{*}, L+\ell\right)}{\beta_{\mathrm{cs}}\left(\varepsilon^{*}, L\right)} \sim\left(\frac{L+\ell}{L}\right)^{-\frac{1}{\nu}}
$$

to the thermodynamic limit. Here it is important to choose $\varepsilon^{*}(L)$ carefully: extrapolating using a series of pseudo-critical points that is close to the gap minimum can yield unreliable results because the derivative of the mass gap remains zero or close to zero. Therefore, we utilize the ratio from the second series of pseudo-critical points $\varepsilon_{L}^{*}=\varepsilon_{b}^{*}(L)$ rather than from the first $\varepsilon_{a}^{*}(L)$ [see Fig. 3(b)]. If $|1 / \nu|<1$ and $L>>\ell$, then

$$
\frac{L}{\ell}\left[\frac{\beta_{\mathrm{cs}}\left(\varepsilon^{*}, L+\ell\right)}{\beta_{\mathrm{cs}}\left(\varepsilon^{*}, L\right)}-1\right] \sim-\frac{1}{\nu} .
$$

From the numerical extrapolation, we obtain

$$
\frac{1}{\nu}=0.996(5)
$$

A plot of unscaled mass-gap data and its collapse using this scaling exponent is shown in Fig. 4.

\section{Thermodynamic exponents $\beta, \alpha, \gamma$}

The bond order parameter characterizes the bondorder-wave (BOW) phase. Fabrizio, Gogolin, and Nersesyan have argued that the bond order parameter is the 


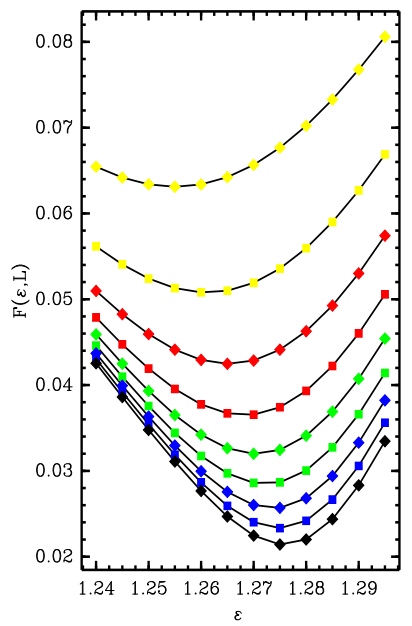

(a)

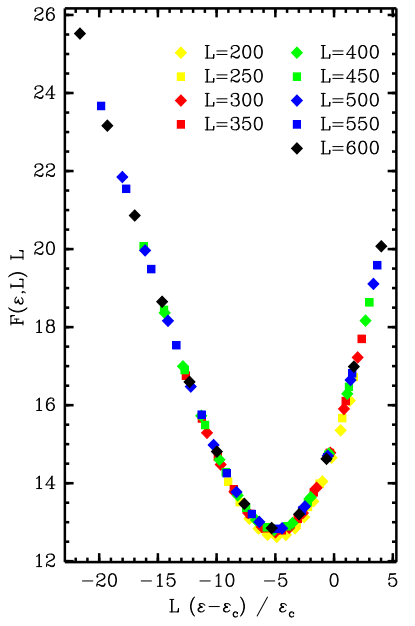

(b)
Figure 4: (Color online) Scaling of the mass gap around the first critical point: (a) unscaled data. The lines are guides to the eye. (b) Rescaled data $F(\varepsilon, L) L$ are plotted as a function of the rescaled coupling $L\left(\varepsilon-\varepsilon_{c_{1}}\right) / \varepsilon_{c_{1}}$.

right quantity to characterize the Ising transition in the IHM $: \underline{14}, \underline{16}$ The order parameter, expressed in the spin-one language, is given by

$$
\begin{gathered}
D(\varepsilon, L)=\frac{1}{L-1} \sum_{i=1}^{L}(-1)^{i}\left[\left\langle\left(\hat{S}_{i}^{+} \hat{S}_{i+1}^{-}+\hat{S}_{i}^{-} \hat{S}_{i+1}^{+}\right) \hat{S}_{i+1}^{z}\right\rangle\right. \\
\left.-\left\langle\hat{S}_{i}^{z}\left(\hat{S}_{i}^{+} \hat{S}_{i+1}^{-}+\hat{S}_{i}^{-} \hat{S}_{i+1}^{+}\right)\right\rangle\right] .
\end{gathered}
$$

DMRG results for the bond order parameter as a function of the coupling $\varepsilon$ near the first transition point are depicted for various system sizes in Fig. 5(a)

We can use the bond order parameter to determine the associated critical exponent $\beta$, i.e.,

$$
D\left(\varepsilon \sim \varepsilon_{c_{1}}, L\right) \sim L^{-\frac{\beta}{\nu}} .
$$

Using the logarithmic derivative

$$
\frac{\ln D\left(\varepsilon^{*}, L+\ell\right)-\ln D\left(\varepsilon^{*}, L\right)}{\ln (L+\ell)-\ln L} \sim-\frac{\beta}{\nu},
$$

we obtain

$$
\frac{\beta}{\nu}=0.124(5) .
$$

The excellent data collapse of the rescaled data, as can be seen in Fig. 5(b), confirms that the transition point belongs to the $2 \mathrm{D}$ Ising universality class. Results for the finite-size scaling of the exponent $\beta$ are plotted in Fig. 6.

Since, in a quantum phase transition, the coupling plays the same role as temperature in a thermal phase

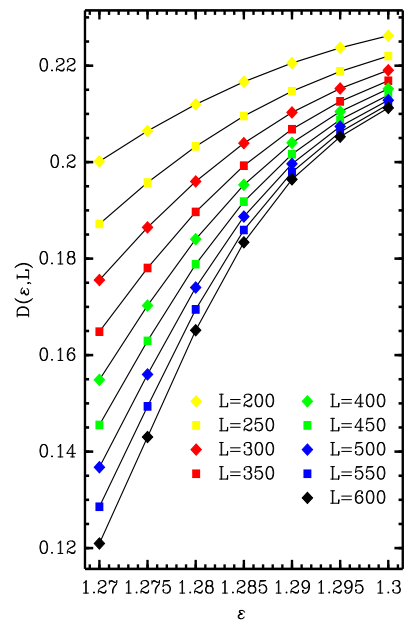

(a)

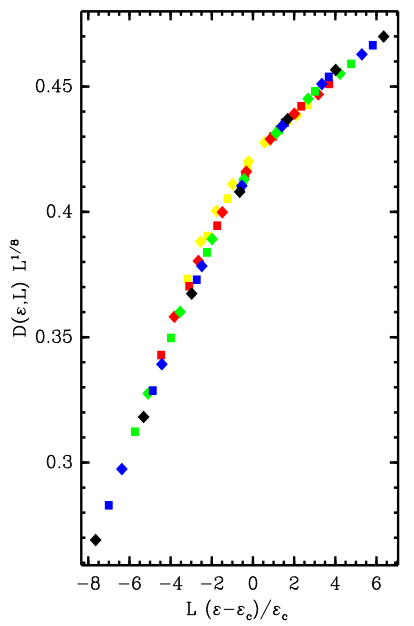

(b)
Figure 5: (Color online) Bond order parameter as a function of the coupling $\varepsilon$ around the transition point: (a) data for different system sizes near the first transition and (b) data rescaled as $D(\varepsilon, L) L^{1 / 8}$ plotted as a function of the rescaled coupling $L\left(\varepsilon-\varepsilon_{c_{1}}\right) / \varepsilon_{c_{1}}$.

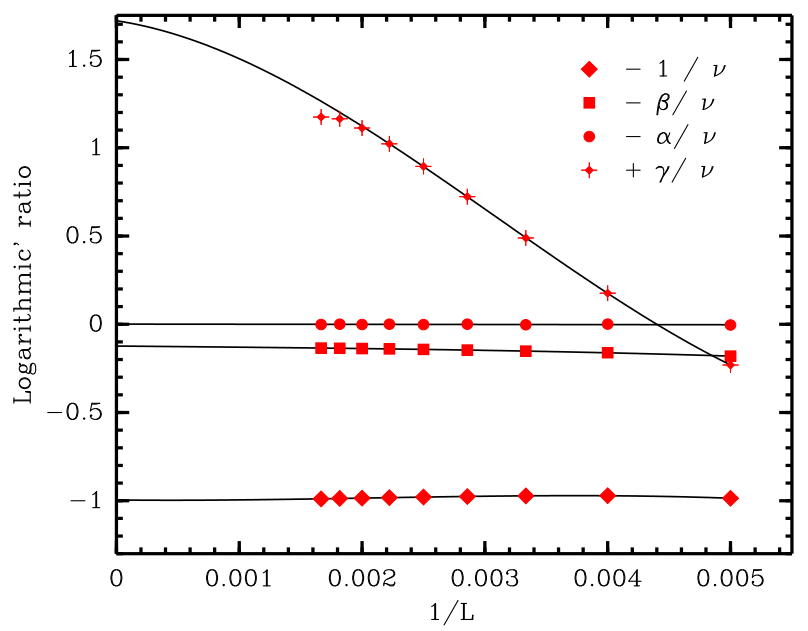

Figure 6: (Color online) Finite-size behavior of the exponents $\nu, \beta, \alpha$, and $\gamma$. The fit is to a third-degree polynomial in $1 / L$. Note that the points for the two smallest $1 / L$ are not included in fitting $\gamma$.

transition, we can define a corresponding "specific heat" $\underline{36.42}$

$$
c_{v}(\varepsilon, L)=-\frac{\varepsilon}{L} \frac{\partial^{2} E_{0}(\varepsilon, L)}{\partial \varepsilon^{2}} .
$$

Note that this quantity does not correspond to the real specific heat. Nevertheless, due to the scaling relations and its interplay with the other quantities, it has to diverge with the exponent $\alpha$. The physical specific heat exponent is related to our $\alpha$ by the Grüneisen parameter $\underline{\underline{43}}$

The specific heat usually contains a regular term that is typically larger in amplitude than the singular one. 
Therefore, instead of using the logarithmic derivative to estimate the exponent $\alpha / \nu$, we instead use the ratio

$$
\frac{L}{2} \frac{c_{v}(\varepsilon, L+2)-c_{v}(\varepsilon, L)}{c_{v}(\varepsilon, L)} \sim \frac{\alpha}{\nu} .
$$

To overcome possible problems in determining this exponent, we use the Hellman-Feynman ${ }^{44}$ theorem to exploit the accuracy of the DMRG in calculating local quantities

$$
\frac{\partial E_{0}(\varepsilon, L)}{\partial \varepsilon}=-\frac{1}{2} \sum_{i}^{L}\left\langle\left(\hat{S}_{i}^{z}\right)^{2}\right\rangle .
$$

This trick reduces the computational cost to that of calculating the first derivative of the cubic spline, which interpolates the data points $\stackrel{45}{=}$ The result is the following:

$$
\frac{\alpha}{\nu}=0.00(1)
$$

The finite-size behavior of the various exponents is plotted in Fig.6. The scaling relation $\alpha=2(1-\nu)$ is fulfilled by Eqs. (14) and (21) 37

Finally, we determine the exponent $\gamma$ associated with the relevant susceptibility. The susceptibility corresponding to the bond order parameter is

$$
\chi_{D}(\varepsilon, L)=-\left.\frac{1}{L} \frac{\partial D(\varepsilon, L)}{\partial h_{D}}\right|_{h_{D}=0} .
$$

In order to calculate this quantity, we turn once more to the Hellman-Feynman theorem and to linear response theory. We perturb the Hamiltonian with a small field $h_{D}$ conjugate to the order parameter $D$. The field has to be small enough to reveal a linear regime in the changes, but not smaller than the actual DMRG resolution; we use $2 \delta h_{D}=10^{-4} t$. We have measured the order parameter for four points around $h_{D}=0$ in order to compute its first derivative at $h_{D}=0$.

Once we have evaluated the static susceptibility for different system sizes, we proceed in the same way as for the previous exponents. The scaling relation is

$$
\chi_{D}\left(\varepsilon_{c_{1}}, L\right) \sim L^{\gamma / \nu} .
$$

Thus, from

$$
\frac{\ln \chi_{D}\left(\varepsilon^{*}, L+\ell\right)-\ln \chi_{D}\left(\varepsilon^{*}, L\right)}{\ln (L+\ell)-\ln L} \sim \frac{\gamma}{\nu}
$$

we obtain the last thermodynamic exponent, as plotted in Fig. 6. with the value

$$
\frac{\gamma}{\nu}=1.72(5)
$$

As shown in the figure, the last points for the largest system sizes have been excluded in calculating the exponent. The reason is that the calculation of the susceptibility becomes uncontrolled for very big system sizes.

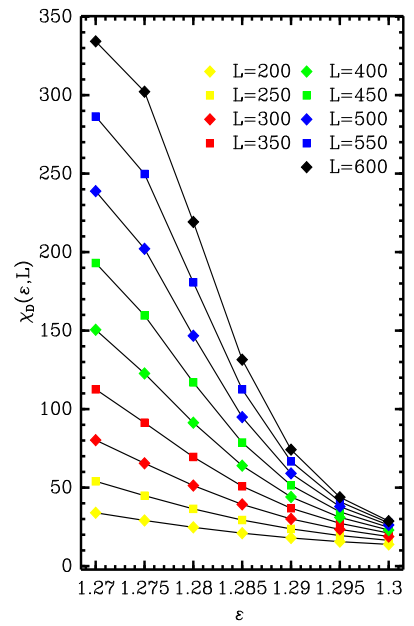

(a)

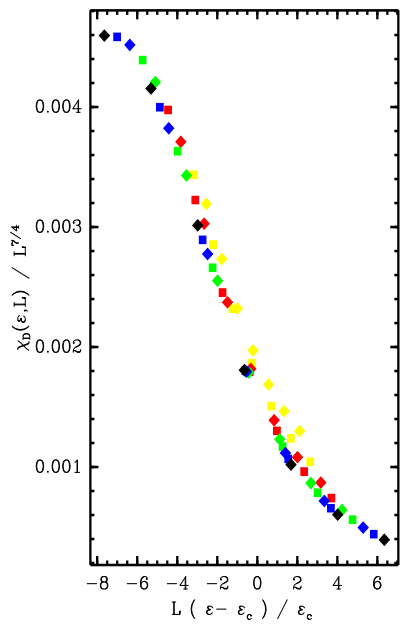

(b)
Figure 7: (Color online) (a) Bond order parameter susceptibility as function of the coupling $\varepsilon$ for different system sizes. (b) The collapsed curves scaled using the exponent $\gamma=7 / 4$.

In order to compensate the occurrence of nonlinear behavior in the response for larger system sizes, we would have to use a very small perturbation field. However, the effect of such a small field can be difficult to distinguish from the numerical noise. In addition, we have to carry out two cubic-spline interpolations: one to determine the derivative of the bond order parameter as function of the perturbation field and one to fit its susceptibility. For these reasons we neglect the points at the two largest system sizes. We see that the second scaling relation $\gamma=2(\nu-\beta)$ is fulfilled to within our estimated error 37 Other quantities, such as the electric polarization and the electric susceptibility, scale with the same exponents as the bond order parameter and the bond-order susceptibility, respectively. 24

In addition, we have calculated the value of the central charge governing the underlying conformal field theory numerically in two different ways. In the first method, we use that the scaling of the low-lying energy levels with system size is uniquely determined by the conformal tower $\underline{46}$ This scaling can be used to determine the central charge .47 The value obtained, $c=0.50(4)$, is consistent with that expected for the $2 \mathrm{D}$ Ising model. In the second method, we determine the central charge from the entropy profile, which has a known form dependent only on the central charge $\stackrel{48}{ }$ We obtain the same value $(c \approx 0.5)$ to within the numerical accuracy at $\varepsilon_{c_{1}}$.

\section{SDI TO MI TRANSITION}

In this section, we present numerical results on the second transition where the system passes from the spontaneously dimerized phase to the Mott insulator phase with increasing $\varepsilon$. We will show both how the spin gap closes when approaching the critical point $\varepsilon_{c_{2}}$ from below and 
how the bond susceptibility diverges when approaching $\varepsilon_{c_{2}}$ from above. Our results confirm the KT scenario with an essential singularity at $\varepsilon_{c_{2}}$ and a critical phase for $\varepsilon>\varepsilon_{c_{2}}$. In order to do this, a more careful treatment than at the 2D Ising transition point is required.

\section{A. Correlation length and mass gap}

For a KT transition, the correlation length diverges exponentially as the transition point is approached from the gapped phase and remains infinite in the critical region that follows $\frac{49}{\underline{4}}$ Since the mass gap is related to the inverse of the correlation length, the mass gap has to close exponentially as the transition is approached and is zero in the critical region. However, for finite-size systems, the correlation length $\xi$ is limited by the system size $L$. Very large system sizes or the inclusion of higher order corrections are required to reveal the exponential divergence, which is restricted to a narrow region close to the KT transition.

In order to locate the position of the second transition point $\varepsilon_{c_{2}}$, we analyze the scaling of the mass gap, depicted in Fig. 8(a) The finite-size scaling analysis made for the first transition cannot be used here because sufficiently large systems to study the logarithmic scaling cannot be reached. Instead, we prefer to use a different approach based on conformal field theory (CFT). Within the Mott insulator phase, where the spin sector is gapless, the system is critical and can be described by a CFT. Furthermore, the characteristic excitation gaps scale with system size $L$ as

$$
E_{i}(L)-E_{0}(L)=\frac{2 \pi x_{i} v}{L},
$$

where $x_{i}$ is the corresponding scaling index and $v$ is the "excitation" velocity. Since this expression is valid only in the critical region corresponding to the Mott insulator, the extent to which it is fulfilled can be used to locate the transition point. In a plot of the mass gap times the system size $L$, Fig. 8(b), all curves merge into a single one exactly at a critical point $\varepsilon_{c_{2}}$, as expected from Eq. (26). Therefore, the system is in a critical regime above a critical coupling

$$
\varepsilon_{c_{2}}=1.8(1) .
$$

The point at which the curves merge is clearly separated, see Eq. (11), from the first critical point that we found at $\varepsilon_{c_{1}}$.

An analysis of the mass gap ratio, see Eq. (10), is also useful. In contrast to what happens at the first transition, $\varepsilon_{c_{1}}$, the curves do not cross the line corresponding to a ratio of unity due to the logarithmic corrections $\underline{41}$ Nevertheless, the curves remain very close to zero everywhere in the critical region above $\varepsilon_{c_{2}}$, as can be seen in Fig. 9(a), In the region preceding $\varepsilon_{c_{2}}$, the value of the mass-gap ratio increases with the system size, as expected for a gapped system. The overall behavior of the

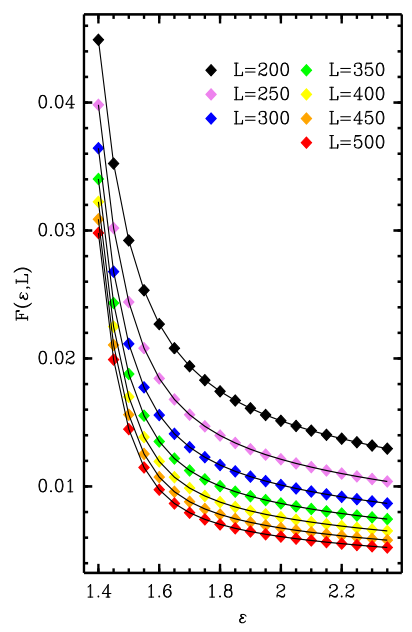

(a)

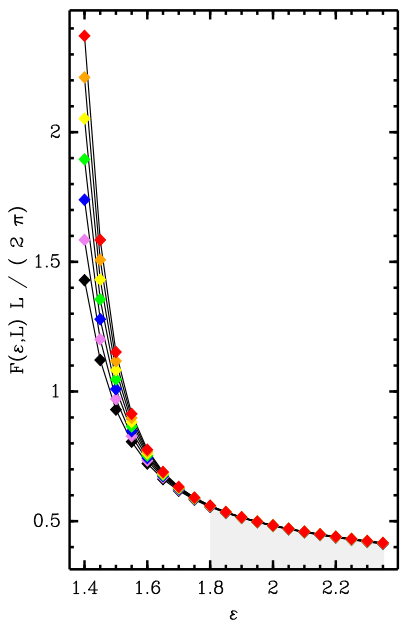

(b)
Figure 8: (Color online) (a) Mass gap and (b) mass gap times $L$ relative to the second critical point as function of the coupling $\varepsilon$ and for different system sizes.

mass-gap ratio curves further confirms that there is a second transition point at $\varepsilon_{c_{2}}$ and supports the KT scenario.

In addition, we define and calculate the scaled difference of mass gaps, $Q$,

$$
Q\left(\varepsilon ; L^{\prime}, L\right)=\frac{L^{\prime}}{2 \pi} \frac{F\left(\varepsilon, L^{\prime}\right) \cdot L^{\prime}-F(\varepsilon, L) \cdot L}{L^{\prime}-L} .
$$

For an arbitrary $L^{\prime}$, the first-order finite-size scaling terms cancel out and $Q\left(\varepsilon ; L^{\prime}, L\right)$ vanishes in the critical region. In Fig. 9(b) we show results for $L^{\prime}=500$. We conclude that the second critical point occurs at $\varepsilon_{c_{2}} \approx 1.8$ and the gap closes exponentially. Since the distance between the two critical points is much bigger, $\varepsilon_{c_{2}}-\varepsilon_{c_{1}} \approx 0.5$, than any deviation due to the logarithmic corrections, we conclude that there are two phase transitions.

We have also calculated the approximate $\beta$-function $\beta_{c s}$, Eq. (12). However, for this kind of transition, it has no zeros (as expected) $\stackrel{41}{\underline{4}}$ Nevertheless, we can extrapolate the value of the minima of the $\beta$-function as a function of the system size to the thermodynamic limit. This yields an alternate estimate of $\varepsilon_{c_{2}}, \varepsilon_{c_{2}}^{\beta}=1.9(1)$.

\section{B. The bond-order and electric susceptibility}

In order to classify the transition as a KT transition, we examine the bond-order susceptibility and the electric susceptibility, see Fig. 10. The behavior of the peak of the bond-order susceptibility can be used to estimate the exponent of the susceptibility,

$$
\gamma_{\text {peak }}^{*}(L)=\frac{\ln \chi\left(\varepsilon_{\text {peak }}^{*}, L+2\right)-\ln \chi\left(\varepsilon_{\text {peak }}^{*}, L\right)}{\ln (L+2)-\ln L} .
$$




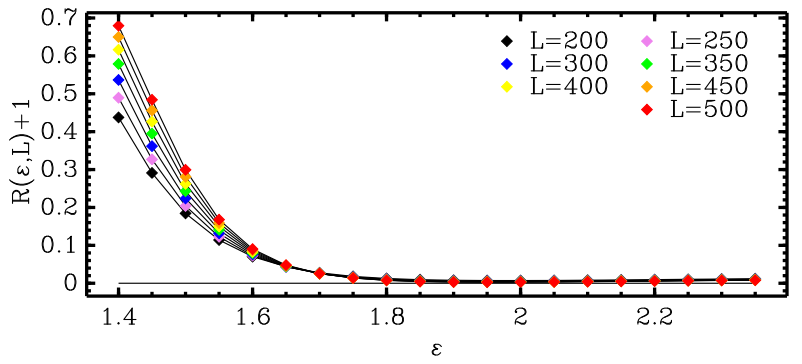

(a)

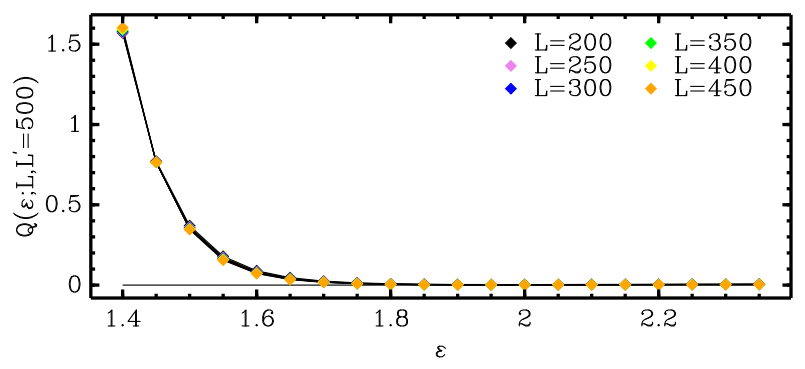

(b)

Figure 9: (Color online) (a) The logarithmic mass gap ratio plus unity. (b) The scaled difference of mass gaps $\mathrm{Q}$ for $L^{\prime}=$ 500 and various values of $L$.

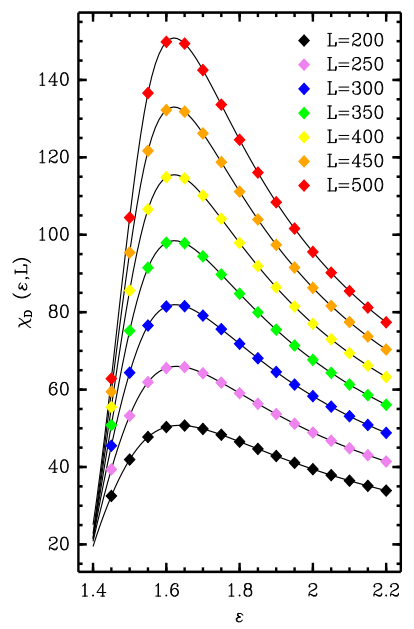

(a)

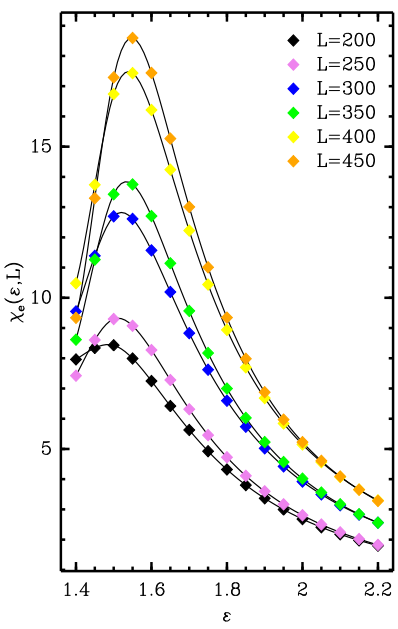

(b)
Figure 10: (Color online) (a) The bond-order susceptibility and (b) the electrical susceptibility for the SDI-MI transition.

The position of the peak in the bond-order susceptibility converges to the value $\varepsilon_{\text {peak }} \approx 1.62$, and the series of pseudo-exponents, $\gamma_{\text {peak }}^{*}(L)$, converges to $\gamma \approx 1.27$ in the thermodynamic limit. For comparison, we calculate the electric susceptibility, shown in Fig. 10(b). The finitesize effects are much stronger for the electric susceptibility than for the bond-order susceptibility. In fact, we also observe a narrow peak in $\chi_{e}$ that grows and moves with the system size. In general, we conclude that the coincidence of the mass gap closing to zero exponentially and a diverging susceptibility corresponds to the typical scenario of an infinite-order phase transition. The critical exponent of the susceptibility $\gamma$ cannot be determined accurately because of strong finite-size effects due to the strong influence of the bond-order wave which scales to zero very slowly, i.e., as $1 / \sqrt{L} \underline{\underline{24}}$

Additionally, we have made a preliminary calculation of the central charge from the entropy profile assuming that it has the form predicted by CFT $\underline{\underline{48}}$ In order to determine the transition point, we minimize the $\chi^{2}$ of the fit to the conformal form and confirm that $c \approx 1$ ( $c=1$ is expected for this type of KT transition) at this point. We obtain a rough estimate of $\varepsilon_{c_{2}}, \varepsilon_{c_{2}}^{c} \approx 1.65(15)$, which is consistent with the results of our finite-size scaling analysis, $\varepsilon_{c_{2}}=1.8(1)$ to within the accuracy of the scaling. Thus, the three estimates of the critical coupling, $\varepsilon_{c_{2}}$, $\varepsilon_{c_{2}}^{\beta}$, and $\varepsilon_{c_{2}}^{c}$ are consistent with one another. Our best estimate is given by $\varepsilon_{c_{2}}$, since the other two estimates are rougher and more likely to contain systematic errors.

\section{DISCUSSION}

We have analyzed the band-insulator-to-Mottinsulator transition in the strong-coupling limit. Using simple strong-coupling arguments, we have derived an effective model starting from the ionic Hubbard model. The effective model, which we have formulated in a spin-one representation, captures the physics of the transition and is less computationally demanding than the ionic Hubbard model. It contains spin-exchange processes which are strongly restricted compared to those of a conventional spin model. In addition, the effective model demonstrates that a single interaction parameter governs the transition. Our density-matrix renormalization group study of this model confirms that there are two transitions at two clearly separated coupling strengths. The system undergoes a transition from a band insulator to a spontaneously dimerized insulator followed by a transition from the spontaneously dimerized phase to a Mott insulator with increasing effective interaction. This behavior corresponds to the behavior of the ionic Hubbard model found in previous work.

In Fig. 11] we explicitly compare the phase boundaries obtained in our work to phase boundaries obtained numerically for the ionic Hubbard model in Refs. 9 and 10. The phase diagram is plotted in the $45^{\circ}$ rotated $U-\Delta$ plane of the ionic Hubbard model, so that the abscissa corresponds to our effective parameter $\varepsilon=U-\Delta$ and the intermediate phase is expanded relative to the depiction in Fig. 1 Since our effective model is based on a strong coupling expansion in $U$ and $\Delta$, our results should be applicable to the ionic Hubbard model in the large $U+\Delta$ limit. As can be seen, for the BI-SDI boundary, both ionic Hubbard model results tend towards our strong-coupling value as $U+\Delta$ becomes larger, although the largest coupling point (at $\Delta=20$ ) from Ref. 10 is still outside our error bars. The results for the SDI-MI 
transition boundary have larger discrepancies, but our results lie between strong $U+\Delta$ extrapolations of the phase boundaries of Ref. 10 and that of Ref. 9. This underlines the difficulty of obtaining the transition point in this infinite-order Kosterlitz-Thouless transition. To within large, but realistic error bars, the three sets of results for this phase boundary are not necessarily inconsistent with each other.

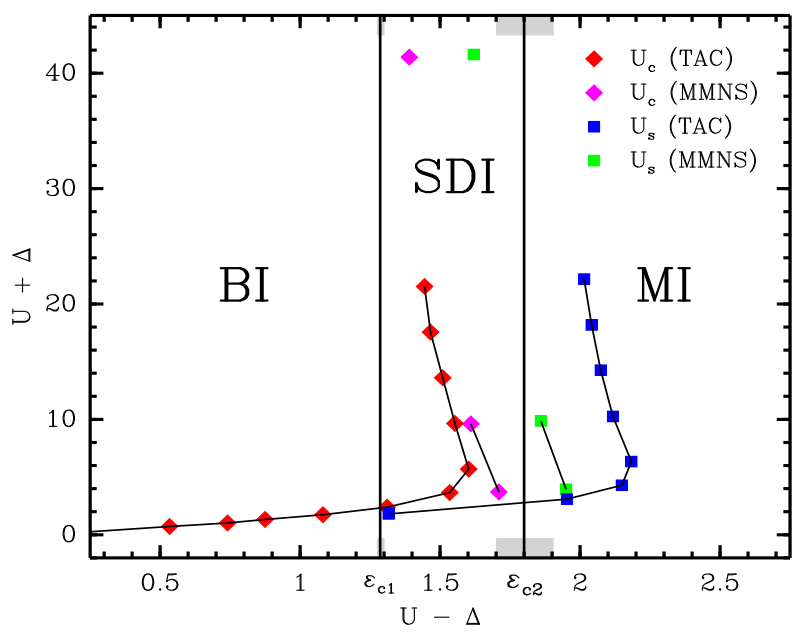

Figure 11: (Color online) Rotated ground-state phase diagram phase diagram of the ionic Hubbard model depicting the phase boundaried obtained in Refs. 9 and 10 , as well as the transition points $\varepsilon_{c_{1}}=1.286(5)$ and $\varepsilon_{c_{2}}=1.8(1)$ obtained in this work, which apply in strong coupling in $U / t, \Delta / t$. The estimated error in our results are indicated by the gray-shaded bars at the upper and lower axes.

Our extraction of the critical exponents for the first transition confirms that it belongs to the two-dimensional Ising universality class. We have also shown that the universal scaling relations are fulfilled to within our numerical accuracy. At the second transition, we have observed that the mass gap closes exponentially and that all relevant susceptibilities diverge. An analysis of the scaling of the mass gap and of the bond-order susceptibility confirms typical Kosterlitz-Thouless behavior. The mass gap closes at the critical point and then remains zero as the interaction is further increased. The susceptibility diverges in the entire critical region above the second transition point. The overall scenario, with an Ising-like transition from the band insulator to the spontaneously dimerized insulator followed by an infinite-order transition from the dimerized insulator the Mott insulator, is in complete agreement with the field-theoretical prediction for the ionic Hubbard model 14

The evolution of the appropriately mapped gaps with increasing $\epsilon$ in the effective model is consistent with the picture obtained for the ionic Hubbard model in Ref. 10. Deep in the band insulating phase, all gaps in the spin and charge sectors are equal and are set by the band gap. As $\varepsilon_{c_{1}}$ is approached, the exciton gap, defined as the energy gap between the ground state and the first singlet excited state, is the mass gap, and it goes to zero at $\varepsilon_{c_{1}}$, while the spin gap (the gap to spin triplet excitations) remains finite. For $\varepsilon_{c_{1}}<\varepsilon<\varepsilon_{c_{2}}$ the mass gap is set by the gap to the lowest-lying triplet, i.e., the spin gap, which is degenerate with singlet excited states. This gap goes to zero at $\varepsilon_{c_{2}}$. For $\varepsilon>\varepsilon_{c_{2}}$, the spin and exciton gaps remain zero, as expected in a critical phase, but the gaps to add or remove one or more particles remain finite.

We note also that the mapping of electronic systems to spin-one systems derived here can be adapted to a larger class of similar models or to generalizations of the ionic Hubbard model, e.g., to chains with an ionic potential with a different periodicity or even to twodimensional systems. Another potentially interesting application would be to relate exactly solvable spin-one models to electronic models and vice versa via the spinone composite representation. 50

\section{Acknowledgments}

L. T. would like to thank B. Normand, G. Japaridze and C. Hamer for useful discussions and the DFG for support through IRTG 790, "Electron-Electron Interactions in Solids". This work was also supported by the Swiss National Foundation through the National Center of Competence in Research "Materials with Novel Electronic Properties-MaNEP".

\section{Appendix: DERIVATION OF THE EFFECTIVE MODEL}

The effective Hamiltonian can most easily be derived by first expressing the original Hamiltonian as a function of the Hubbard operators $\hat{X}_{i}^{\alpha \beta}=\left|\alpha_{i}\right\rangle\left\langle\beta_{i}\right|$, where the $\left|\alpha_{i}\right\rangle$ and $\left|\beta_{i}\right\rangle$ designate an element of the Hubbard basis $\{|0\rangle,|\uparrow\rangle,|\downarrow\rangle,|d\rangle\}$ on site $i$. Explicitly, they can be expressed as

$$
\hat{X}=\left[\begin{array}{cccc}
\left(\hat{1}-\hat{n}_{\downarrow}\right)\left(\hat{1}-\hat{n}_{\uparrow}\right) & \hat{c}_{\uparrow}\left(\hat{1}-\hat{n}_{\downarrow}\right) & \hat{c}_{\downarrow}\left(\hat{1}-\hat{n}_{\uparrow}\right) & \hat{c}_{\downarrow} \hat{c}_{\uparrow} \\
\hat{c}_{\uparrow}^{\dagger}\left(\hat{1}-\hat{n}_{\downarrow}\right) & \left(\hat{1}-\hat{n}_{\downarrow}\right) \hat{n}_{\uparrow} & \hat{c}_{\uparrow}^{\dagger} \hat{c}_{\downarrow} & -\hat{c}_{\downarrow} \hat{n}_{\uparrow} \\
\hat{c}_{\downarrow}^{\dagger}\left(\hat{1}-\hat{n}_{\uparrow}\right) & \hat{c}_{\downarrow}^{\dagger} \hat{c}_{\uparrow} & \hat{n}_{\downarrow}\left(\hat{1}-\hat{n}_{\uparrow}\right) & \hat{n}_{\downarrow} \hat{c}_{\uparrow} \\
\hat{c}_{\uparrow}^{\dagger} \hat{c}_{\downarrow}^{\dagger} & -\hat{c}_{\downarrow}^{\dagger} \hat{n}_{\uparrow} & \hat{n}_{\downarrow} \hat{c}_{\uparrow}^{\dagger} & \hat{n}_{\downarrow} \hat{n}_{\uparrow}
\end{array}\right] .
$$

For instance, we rewrite the ionic potential and the Coulomb interaction as

$$
\begin{aligned}
\hat{H}_{U} & =U \sum_{i=1}^{L} \hat{X}_{i}^{d d} \\
& =U \sum_{j=1}^{L / 2}\left(\hat{X}_{2 j-1}^{d d}+\hat{X}_{2 j}^{d d}\right)
\end{aligned}
$$


and

$$
\begin{aligned}
& \hat{H}_{\Delta}= \frac{\Delta}{2} \sum_{i=1}^{L}(-1)^{i}\left(\hat{X}_{i}^{\uparrow \uparrow}+\hat{X}_{i}^{\downarrow \downarrow}+2 \hat{X}_{i}^{d d}\right) \\
&= \frac{\Delta}{2} \sum_{j=1}^{L / 2}\left(-\hat{X}_{2 j-1}^{\uparrow \uparrow}-\hat{X}_{2 j-1}^{\downarrow \downarrow}-2 \hat{X}_{2 j-1}^{d d}\right. \\
&\left.+\hat{X}_{2 j}^{\uparrow \uparrow}+\hat{X}_{2 j}^{\downarrow \downarrow}+2 \hat{X}_{2 j}^{d d}\right) .
\end{aligned}
$$

They can then be mapped onto the spin-one model expressed in terms of the operators $L_{i}^{s s^{\prime}}=\left|s_{i}\right\rangle\left\langle s_{i}^{\prime}\right|$, with $\left|s_{i}\right\rangle$ the spin-one $S_{z}$ basis $\{|1\rangle,|0\rangle,|-1\rangle\}$ on site $i$. The single-site Hilbert space truncation is defined as

$$
\begin{cases}\hat{X}_{i}^{\alpha \beta} \rightarrow 0 & \text { for } \alpha \text { or } \beta=0 \text { and } i=2 j-1 \\ \hat{X}_{i}^{\alpha \beta} \rightarrow 0 & \text { for } \alpha \text { or } \beta=d \text { and } i=2 j \\ \hat{X}_{i}^{\alpha \beta}=\hat{L}^{\alpha \beta} & \text { otherwise. }\end{cases}
$$

In the spin-one basis,

$$
\hat{L}=\left[\begin{array}{ccc}
\frac{\left(\hat{S}_{i}^{z}\right)^{2}+\hat{S}_{i}^{z}}{2} & \frac{\hat{S}_{i}^{z} \hat{S}_{i}^{+}}{\sqrt{2}} & \frac{\left(\hat{S}_{i}^{+}\right)^{2}}{2} \\
\frac{\hat{S}_{i}^{-} \hat{S}_{i}^{z}}{\sqrt{2}} & \hat{1}_{i}-\left(\hat{S}_{i}^{z}\right)^{2} & -\frac{\hat{S}_{i}^{+} \hat{S}_{i}^{z}}{\sqrt{2}} \\
\frac{\left(\hat{S}_{i}^{-}\right)^{2}}{2} & -\frac{\hat{S}_{i}^{z} \hat{S}_{i}^{-}}{\sqrt{2}} & \frac{\left(\hat{S}_{i}^{z}\right)^{2}-\hat{S}_{i}^{z}}{2}
\end{array}\right] .
$$

Hence, the interaction and the potential parts are transformed to

$$
\begin{gathered}
\hat{H}_{U}=U \sum_{j=1}^{L / 2} \hat{L}_{2 j-1}^{00}, \\
\hat{H}_{\Delta}=-\frac{\Delta}{2} \sum_{j=1}^{L / 2}\left(\hat{L}_{2 j-1}^{11}+\hat{L}_{2 j-1}^{-1-1}+2 \hat{L}_{2 j-1}^{00}\right. \\
\left.-\hat{L}_{2 j}^{11}-\hat{L}_{2 j}^{-1-1}\right) .
\end{gathered}
$$

Altogether, defining the coupling constant $\varepsilon=U-\Delta$, the doping $\delta=N-L$, and writing the terms using spinone operators, see Eq. A.2 , the two-term contribution becomes

$$
\hat{H}_{\varepsilon}^{e}=-\frac{\varepsilon}{2} \sum_{i=1}^{L}\left(\hat{S}_{i}^{z}\right)^{2}-\frac{\varepsilon}{2} L-\frac{U}{2} \delta .
$$

Likewise, the hopping part is translated to

$$
\begin{array}{r}
\hat{H}_{t}^{e}=t \sum_{i=1}^{L}\left(\hat{L}_{i}^{0-1} \hat{L}_{i+1}^{01}-\hat{L}_{i}^{01} \hat{L}_{i+1}^{0-1}\right. \\
\left.+\hat{L}_{i}^{-10} \hat{L}_{i+1}^{10}-\hat{L}_{i}^{10} \hat{L}_{i+1}^{-10}\right)
\end{array}
$$

or, in the spin one language

$$
\begin{aligned}
\hat{H}_{t}^{e}=\frac{t}{2} \sum_{i=1}^{L} & \left(-\hat{S}_{i}^{+} \hat{S}_{i}^{z} \hat{S}_{i+1}^{-} \hat{S}_{i+1}^{z}\right. \\
& \left.+\hat{S}_{i}^{-} \hat{S}_{i}^{z} \hat{S}_{i+1}^{+} \hat{S}_{i+1}^{z}+\text { h.c. }\right),
\end{aligned}
$$

which is equivalent to Eq. (5). A sketch of the allowed processes is shown in Fig. 12] These processes are a relative small subset of those of the isotropic Heisenberg spin chain model. Note that the AFM exchange in the IHM maps to a sequence consisting of two scattering processes in the effective model.

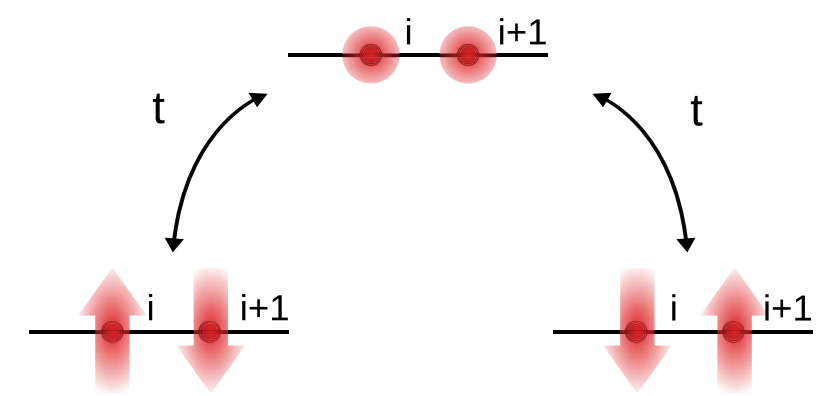

Figure 12: (Color online) Sketch of the allowed processes, which are a relatively small subset of those of the isotropic Heisenberg spin-chain model.
* leonildo.tincani@physik.uni-marburg.de

1 N. Nagaosa and J. Takimoto, J. Phys. Soc. Jpn. 55, 2735 (1986).

2 N. Nagaosa and J. Takimoto, J. Phys. Soc. Jpn. 55, 2745 (1986).

3 N. Nagaosa, J. Phys. Soc. Jpn. 55, 2754 (1986).

4 J. B. Torrance, J. E. Vazquez, J. J. Mayerle, and V. Y. Lee, Phys. Rev. Lett. 46, 253 (1981).

5 S. Horiuchi, Y. Okimoto, R. Kumai, and Y. Tokura, Science 299, 229 (2003).

6 J. T. Gammel, A. Saxena, I. Batistić, A. R. Bishop, and S. R. Phillpot, Phys. Rev. B 45, 6408 (1992).
7 S. Yamamoto, Phys. Rev. B 63, 125124 (2001).

${ }^{8}$ F. H. L. Essler, H. Frahm, F. Göhmann, A. Klümper, and V. E. Korepin, The One-Dimensional Hubbard Model (Cambridge University Press, 2005).

9 M. E. Torio, A. A. Aligia, and H. A. Ceccatto, Phys. Rev. B 64, 121105 (2001).

10 S. R. Manmana, V. Meden, R. M. Noack, and K. Schönhammer, Phys. Rev. B 70, 155115 (2004).

11 A. P. Kampf, M. Sekania, G. I. Japaridze, and P. Brune, J. Phys. : Condens. Matter 15, 5895 (2003).

12 P. Kakashvili and G. Japaridze, J. Phys. C: Solid State Phys. 16, 5815 (2004). 
13 R. Resta and S. Sorella, Phys. Rev. Lett. 74, 4738 (1995).

14 M. Fabrizio, A. O. Gogolin, and A. A. Nersesyan, Phys. Rev. Lett. 83, 2014 (1999).

15 G. Delfino and G. Mussardo, Nucl. Phys. B 516, 674 (1998).

16 M. Fabrizio, A. O. Gogolin, and A. A. Nersesyan, Nucl. Phys. B 580, 647 (2000).

17 N. Gidopoulos, S. Sorella, and E. Tosatti, Eur. Phys. J. B 14, 217 (2000).

18 T. Wilkens and R. M. Martin, Phys. Rev. B 63, 235108 (2001).

19 J. Lou, S. Qin, T. Xiang, C. Chen, G.-S. Tian, and Z. Su, Phys. Rev. B 68, 045110 (2003).

20 H. Otsuka and M. Nakamura, Phys. Rev. B 71, 155105 (2005).

21 Ö. Legeza and J. Sólyom, Phys. Rev. Lett. 96, 116401 (2006).

22 Ö. Legeza, K. Buchta, and J. Sólyom, Phys. Rev. B 73, 165124 (2006).

23 A. A. Aligia and C. D. Batista, Phys. Rev. B 71, 125110 (2005).

${ }^{24}$ L. Tincani, Ph.D. thesis, University of Marburg (2008).

25 P. Fazekas, Lecture Notes on Electron Correlation and Magnetism (World Scientific, 1999).

26 S. B. Haley and P. Erdös, Phys. Rev. B 5, 1106 (1972).

27 B. Horovitz and J. Sólyom, Phys. Rev. B 35, 7081 (1987).

28 S. R. White, Phys. Rev. Lett. 69, 2863 (1992).

29 U. Schollwöck, Rev. Mod. Phys. 77, 259 (2005).

${ }^{30}$ M. Fabrizio and A. O. Gogolin, Phys. Rev. B 51, 17827 (1995).

31 G. Bedürftig, B. Brendel, H. Frahm, and R. M. Noack, Phys. Rev. B 58, 10225 (1998).

32 C. Hotta and N. Shibata, Physica B 378, 1039 (2006).
33 Ö. Legeza and G. Fáth, Phys. Rev. B 53, 14349 (1996).

34 Ö. Legeza, J. Röder, and B. A. Hess, Phys. Rev. B 67, 125114 (2003).

35 Ö. Legeza and J. Sólyom, Phys. Rev. B 68, 195116 (2003).

36 J. B. Kogut, Rev. Mod. Phys. 51, 659 (1979).

37 M. N. Barber, in Phase Transition and Critical Phenomena, edited by C. Domb and M. S. Green (Academic Press, 1983), vol. 8 of Phase Transition and Critical Phenomena, chap. 2.

38 U. Glaus and T. Schneider, Phys. Rev. B 30, 215 (1984).

39 C. J. Hamer and M. N. Barber, J. Phys. A: Math. Gen. 13, L169 (1980).

${ }^{40}$ C. J. Hamer and M. N. Barber, J. Phys. A: Math. Gen. 14, 241 (1981).

41 C. J. Hamer and M. N. Barber, J. Phys. A: Math. Gen. 14, 259 (1981).

42 C. J. Hamer, J. Phys. A: Math. Gen. 16, 3085 (1983).

43 L. Zhu, M. Garst, A. Rosch, and Q. Si, Phys. Rev. Lett. 91, 066404 (2003).

44 R. P. Feynman, Phys. Rev. 56, 340 (1939).

${ }^{45}$ W. H. Press, B. P. Flannery, S. A. Teukolsky, and W. T. Vetterling, Numerical Recipes in $\mathrm{C}++$, The Art of Scientific Computing (Cambridge University Press, 1999).

46 C. Degli Esposti Boschi and F. Ortolani, Eur. Phys. J. B 41, 503 (2004).

47 P. Di Francesco, P. Mathieu, and D. Sénéchal, Conformal Field Theory (Springer, 1999).

48 P. Calabrese and J. Cardy, J. Stat. Mech.: Theor. Exp. 2004, P06002 (2004).

49 J. M. Kosterlitz, J. Phys. C: Solid State Phys. 7, 1046 (1974).

50 C. D. Batista and G. Ortiz, Adv. Phys. 53, 1 (2004). 